\title{
'a'-Position-Mutated and G4-Mutated Hemagglutinin-Neuraminidase Proteins of Newcastle Disease Virus Impair Fusion and Hemagglutinin-Neuraminidase-Fusion Interaction by Different Mechanisms
}

\author{
Fu-lu Chu ${ }^{a}$ Hong-ling Wen ${ }^{a}$ Wen-qiang Zhang ${ }^{c}$ Bin Lin ${ }^{c}$ Yan Zhang ${ }^{c}$ \\ Cheng-xi Sun $^{a}$ Gui-jie Ren $^{b}$ Yan-yan Song ${ }^{a}$ Zhiyu Wang ${ }^{a}$ d \\ a Department of Virology, School of Public Health and ${ }^{b}$ Institute of Biochemistry and Molecular Biology, \\ School of Medicine, Shandong University, ' Shandong Center for Disease Control and Prevention, and \\ ${ }^{\mathrm{d}}$ The Key Laboratory for Experimental Teratology of the Ministry of Education, Jinan, China
}

\section{Key Words}

Paramyxovirus - Hemagglutinin-neuraminidase protein • Heptad repeat region - Glycosylation - Membrane fusion • Hemagglutinin-neuraminidase-fusion protein complexes

\begin{abstract}
Objectives: To determine the effects of heptad repeat regions (HRs) and $\mathrm{N}$-linked carbohydrate sites of the Newcastle disease virus hemagglutinin-neuraminidase $(\mathrm{HN})$ protein on fusion of $\mathrm{HN}$ and fusion (F) proteins and HN-F interaction. Methods: We mutated six 'a' residues in the HRs and four asparagines in $\mathrm{N}$-linked carbohydrate sites to alanine in the $\mathrm{HN}$ protein. A vaccinia-T7 RNA polymerase expression system was used to express HN cDNAs in BHK-21 cells to determine the HN functions. Deglycosylation was treated with PGNase $\mathrm{F}$ digestion. The formation of HN-F protein complexes was determined by the coimmunoprecipitation assay. Results: Each HR-mutated protein interfered with fusion and the $\mathrm{HN}-\mathrm{F}$ interaction. The G4-mutated protein not only impaired fusion and $\mathrm{HN}-\mathrm{F}$ interaction but also decreased activities in cell fusion promotion, hemadsorption and neuraminidase. Conclusions: It is assumed that two different mechanisms
\end{abstract}

for mutations in these two regions are responsible for the decreased fusion promotion activity and the reduced ability of interaction with $\mathrm{F}$ protein. Mutations in the HRs impair fusion and $\mathrm{HN}-\mathrm{F}$ interaction by altering the transmission of a signal from the globular domain to the F-specific region in the stalk, but the G4 mutation modulates fusion and HN-F interaction by the misfolding of some important structures.

Copyright $\odot 2012$ S. Karger AG, Basel

\section{Introduction}

Newcastle disease virus (NDV) belongs to the family Paramyxoviridae, a group of enveloped viruses with nonsegmented, negative-sense, single-stranded RNA genome [1]. It causes a series of severe respiratory and neurological diseases in all species of birds worldwide [2-4], but there is no effective vaccine for preventing the disease [4].

NDV enters host cells by fusion of the viral and cellular membranes. The envelope of NDV contains two glycoproteins, the hemagglutinin-neuraminidase $(\mathrm{HN})$ and the fusion $(\mathrm{F})$ proteins, which are modified by the covalent

\section{KARGER \\ Fax +4161306 1234 \\ E-Mail karger@karger.ch}

www.karger.com (c) 2012 S. Karger AG, Basel

$0300-5526 / 13 / 0561-0027 \$ 38.00 / 0$

Accessible online at:

www.karger.com/int
Dr. Zhiyu Wang

Department of Virology, School of Public Health

Shandong University, 44 Wen-hua-xi-lu Road

Jinan 250012 (China)

Tel. +86 5318838 0418, E-Mail zhiyu.wang@ @du.edu.cn 
attachment of N-linked oligosaccharide chains. NDV HN recognizes and binds to sialic acid-containing receptors on cell surfaces [hemadsorption (HAD) activity]. HN also acts as a neuraminidase (NA) by removing the sialic acid from progeny virus particles to prevent self-agglutination (NA activity) [5, 6]. NDV F protein mediates fusion of the virus membrane and the cell membrane, resulting in virus penetration of the cell surface. Optimum membrane fusion (cell fusion promotion activity) requires both NDV $\mathrm{F}$ protein and HN protein. Therefore, some researchers proposed that a type-specific interaction between the paramyxovirus $\mathrm{HN}$ and $\mathrm{F}$ proteins is necessary for fusion of virus with cell membrane [7-10].

However, the domain of the $\mathrm{HN}$ protein that interacts with the F protein is not understood. Previous studies of chimeric $\mathrm{HN}$ proteins revealed that the putative stalk domain of paramyxoviruses is important for interaction with $F$ protein and promotion of cell fusion [11-13]. Heptad repeat regions (HRs) are often involved in proteinprotein interactions and could form alpha helices [14]. The NDV HN protein has two characterized HRs in the stalk domain, HR1 and HR2 [14, 15]. Mutational analyses of the HRs of paramyxoviruses $\mathrm{F}$ proteins have shown that they are important in fusion activity as well as the folding of their F proteins [16-18]. Other studies showed that substitutions at the heptadic positions played an important role in the functions of NDV HN protein. However, the effect of these substitutions on the HN-F interaction was not determined [14, 19-21].

$\mathrm{N}$-linked glycosylation has some influence on the correct folding of $\mathrm{HN}$ into its biologically active conformation and protein activity. A carbohydrate link site in close proximity to one of the HRs could affect the structure and function of this domain [22]. Addition of N-glycans in the HRs of the HN protein blocks its interaction with the F protein and prevents fusion [23]. NDV HN protein has six potential $\mathrm{N}$-linked glycosylation sites, only four (G1-G4 at residues 119, 341, 433, and 481, respectively) of which are utilized [23]. Loss of $\mathrm{N}$-linked glycosylation from $\mathrm{HN}$ protein altered the functions of $\mathrm{HN}$ protein and the virulence of NDV. But the effect of elimination of Nlinked carbohydrate addition sites on the HN-F interaction was not determined $[24,25]$.

In this study we performed site-directed mutational analyses of the HRs in the HN protein from the NDV and quantified HN-F protein complexes to determine the effect of substitutions on the HN-F interaction. We also characterized the effect of the mutation individually of four glycosylation addition sites of NDV HN and detected HN-F protein complexes by coimmunoprecipitation (co-IP).

\section{Materials and Methods}

Recombinant Plasmids and Site-Directed Mutagenesis

The wild-type (wt) or mutated HN genes were inserted into pBluescript SK (+) $\left(\mathrm{pBSK}^{+}\right)$at the Xba I and Sac I sites and the F gene at the Xho I site. Escherichia coli TG1 was cultured in $2 \times$ YT medium. Recombinant plasmids were purified from TG1 cells by using ENZA Plasmid Miniprep Kit (Omega Bio-Tek, Inc., Norcross, Ga., USA). Site-directed mutagenesis was performed as described previously [26], using NDV HN vector as the template and two pairs of reverse-complement mutagenesis primers (Sangon Biotech Co. Ltd., Shanghai, China) (suppl. material tables 1 and 2). Two PCR products with homologous ends were transformed into TG1 cells and then recombined to a complete plasmid containing the mutated $\mathrm{HN}$ gene (suppl. material figures $1 \mathrm{a}$ and $1 \mathrm{~b}$ ). The presence of the desired mutation was confirmed by DNA sequencing (suppl. material figures 4 and 5).

Eukaryotic Transient-Expression System and Quantitation of Cell Surface Expression

BHK-21 cells was provided by Shandong Academy of Medical Sciences and were cultured in Dulbecco's modified Eagle's medium (Gibco) with high glucose, L-glutamine, and pyridoxine hydrochloride supplemented with $10 \%$ fetal calf serum (FCS; Gibco). wt and mutated HN proteins were expressed in BHK-21 cells, respectively, using the vaccinia virus-T7 (vTF7-3) RNA polymerase expression system [27]. All experiments, except for the NA assays, were performed in 6 -well plates seeded a day earlier at $4 \times$ $10^{5}$ cells per well. Maintenance of the cells, infection with vTF7-3, and Lipofectamine ${ }^{\mathrm{TM}} 2000$ (Invitrogen) transfections were performed as described previously [28], using $1 \mu \mathrm{g}$ of each plasmid for transfection. Cell surface expression (CSE) was quantitated by fluorescence-activated cell sorter (FACS) with a mixture of two mouse monoclonal antibodies (MAbs) in the HN globular domain (dilution 1:100), including HN 4a and HN IC114 (Chemicon International, Inc., Temecula, Calif., USA). The cells were washed with phosphate-buffered saline containing 5\% FCS (PBS-FCS) and then incubated with a 1:200 dilution of fluorescein isothiocyanate conjugated to goat anti-mouse IgG (ZSGB-BIO, Beijing, China) in PBS-FCS for $30 \mathrm{~min}$. After two additional washes with PBS-FCS, the monolayers were detached with $0.0625 \mathrm{mM}$ EDTA in PBS, pelleted by centrifugation, and washed once with PBSFCS. The cells were fixed in PBS with $1 \%$ FCS plus $1 \%$ paraformaldehyde prior to analysis. The cell surface fluorescence of 10,000 cells was measured using a FACSCalibur flow cytometer (Becton Dickinson). Medium fluorescence intensities were normalized to the medium fluorescence intensities of the NDV wt HN protein. Cells transfected with vector alone and incubated with both primary and secondary antibodies were used as negative controls $[15,21,29]$.

\section{HAD Assay}

The HAD activity of $\mathrm{HN}$ proteins was assayed by the ability of the transfected cells absorbing guinea pig erythrocytes. The monolayers that expressed HN proteins were incubated for 30 $\min$ at $4^{\circ}$ with a $2 \%$ suspension of guinea pig erythrocytes in PBS supplemented with $0.1 \mathrm{mM} \mathrm{CaCl}_{2}$ and $1 \mathrm{mM} \mathrm{MgCl}_{2}$ (PBS-CM). Absorbed erythrocytes were lysed in $50 \mathrm{mM} \mathrm{NH}_{4} \mathrm{Cl}$ and the lysates were clarified by centrifugation. The absorbance of the samples was measured at 540-nm wavelength on the UV spectropho- 
tometer (Shimadzu, Kyoto, Japan) minus background absorbance of cells expressing the vector alone $[15,21,29]$.

\section{NA Assay}

NA assay was performed on 22.6-mm-diameter plates seeded 1 day earlier with $1.6 \times 10^{5}$ cells/well and transfected with $0.5 \mu \mathrm{g}$ of DNA. To each well was added $0.5 \mathrm{ml}$ of $625 \mu \mathrm{g} / \mathrm{ml}$ of neuraminlactose (Sigma) in $0.1 \mathrm{M}$ sodium acetate ( $\mathrm{pH}$ 6.0) and the plates were incubated at $37^{\circ}$ for $1 \mathrm{~h}$. NA activity was quantitated by measuring the intensities of the colors in the butanol layer at $549 \mathrm{~nm}$ minus the background of cells expressing the vector alone $[15,21$, $29,30]$.

\section{Assay for Cell Fusion}

The ability of HN proteins to promote fusion was quantified by a modification of the reporter gene method described previously [20,29]. Briefly, monolayers of BHK-21 effector cells seeded 1 day earlier with $4 \times 10^{5}$ cells $/ 35-\mathrm{mm}$ plate were infected with the recombinant vTF7-3 vaccina and cotransfected with the desired $\mathrm{HN}$ and $\mathrm{F}$ genes. Monolayers of BHK-21 target cells were infected with wt vaccinia (m.o.i. $=10)$ and transfected with $1 \mu \mathrm{g}$ of plasmid pG1NT7 $\beta$-gal per well, which carries the $\beta$-galactosidase gene under the control of a T7 promoter.

From 20 to $22 \mathrm{~h}$ after transfection, cells were removed from the wells by treatment with $0.05 \%$ trypsin and $0.53 \mathrm{~mm} \mathrm{EDTA}$ (Gibco) and washed with Dulbecco's modified Eagle's medium. Equal numbers $\left(1 \times 10^{5}\right)$ of the recombinant vaccina and wt vaccineinfected cells were combined in duplicate wells of a 96 -well microtiter plate. After incubation at $37^{\circ}$ for $5 \mathrm{~h}$, the cells were lysed with $50 \mu \mathrm{l}$ of $0.25 \% \mathrm{NP}-40$ at room temperature for $30 \mathrm{~min}$. Then, $20 \mu \mathrm{l}$ of supernatant from each well was withdrawn and mixed with $130 \mu$ l of the $\beta$-galactosidase substrate $1.2 \mathrm{mg} / \mathrm{ml}$ chlorophenol red- $\beta$-D-galactopyranoside. After incubation for $20 \mathrm{~min}$ at room temperature, the extent of fusion was quantitated by determination of the absorbance at $590 \mathrm{~nm}$ with an automated ELISA reader by subtracting the background of the cells expressing NDV F alone.

Immunoprecipitation and Peptidyl-N-Glycosidase F Digestion The immunoprecipitation (IP) protocol was described previously [31]. Briefly, at $22 \mathrm{~h}$ posttransfection, BHK-21 cells were starved for $1 \mathrm{~h}$ at $37^{\circ}$ in medium lacking cysteine and methionine. The cells were then labeled with $1 \mathrm{ml}$ of medium containing $100 \mu \mathrm{Ci}$ of Expre ${ }^{35} \mathrm{~S}^{35} \mathrm{~S}$ labeling mix (Perkin-Elmer Life and Analytical Sciences, Boston, Mass., USA) for $3 \mathrm{~h}$ at $37^{\circ}$ and chased for $90 \mathrm{~min}$ with nonradioactive medium. The cells were lysed by PBS containing 1\% Triton X-100, 0.5\% deoxycholate, and $1 \mathrm{mM}$ phenylmethylsulfonyl fluoride (PMSF). The HN proteins were immunoprecipitated with a mixture of two MAbs containing $\mathrm{HN} 4 \mathrm{a}$ and $\mathrm{HN}$ IC114 which were cross-linked to Dynabeads protein A (Invitrogen). The Dynabeads MAbs proteins were washed 3 times with PBS using a magnet. Proteins were eluted by boiling in protein loading buffer and then analyzed by $10 \%$ sodium dodecyl sulfate-polyacrylamide gel electrophoresis (SDS-PAGE).

For peptidyl-N-glycosidase (PNGase) F digestion, the Dynabeads MAbs proteins were resuspended in PNGase denaturation buffer containing $0.5 \%$ SDS and boiled for $5 \mathrm{~min}$. The solution was allowed to cool and adjusted to contain $0.1 \%$ SDS and $0.5 \%$ NP-40. One aliquot of each sample was digested with $200 \mathrm{mU}$ of
PNGase F (New England Biolabs, Beverly, Mass., USA) for $16 \mathrm{~h}$ at $37^{\circ}$ prior to SDS-PAGE under reducing conditions $[28,31]$.

\section{Co-IP Assay}

The ability of wt and mutated $\mathrm{HN}$ proteins to interact with $\mathrm{F}$ at the surface of transfected BHK-21 cells was assayed at $22 \mathrm{~h}$ using the co-IP assay described previously $[15,20,21]$. Because the amount of the $\mathrm{F}$ protein immunoprecipitated from the cell surface is greatly affected by the extent of fusion, a cleavage site mutant form of $\mathrm{F}$ (Fcsm) that does not fuse, but interacts efficiently with the $\mathrm{HN}$ protein, was used. For this assay, equal numbers of cells were starved and labeled as described previously. Cells were washed 3 times with cold PBS-CM. Cell surface proteins were biotinylated with sulfo-NHS-SS-biotin (Pierce, Rockford, Ill., USA) for $30 \mathrm{~min}$ at $4^{\circ}$ with gentle agitation, followed by removal of the excess reagent by washing twice with PBS-CM. The labeled cells were lysed by PBS containing $1 \%$ Triton X-100, 0.5\% deoxycholate, and $1 \mathrm{mM}$ PMSF for $45 \mathrm{~min}$ on ice. Lysates from 3 wells of a 6 -well plate were combined and split into two equal aliquots into tubes containing Dynabead-cross-linked antibodies (either an MAb specific for the F protein only or this MAb plus two anti-HN MAbs). The anti-F antibody was purchased from Changzhou AbBioSci Inc. The IP reaction mixes were incubated for $30 \mathrm{~min}$ at room temperature and then washed 3 times with PBS using a magnet. Bound proteins were released by boiling for $5 \mathrm{~min}$ in $10 \mu \mathrm{l}$ of $10 \%$ SDS and were resuspended in PBS. Proteins were precipitated from the supernatant with streptavidin beads (Pierce) overnight at $4^{\circ}$. The beads were washed twice with PBS and then resuspended in SDS-PAGE loading buffer. The percentage of total cell surface $\mathrm{HN}$ that co-IP with F was quantified with a Perkin Elmer Cyclone Plus. The cells expressing vector, HN, or Fcsm alone were used as controls to ensure that the co-IP of HN by the F antibody occurred only through its interaction with the $\mathrm{F}$ protein.

\section{Results}

Alanine-Scanning Mutagenesis of the HRs and the Glycosylation Sites of the NDV HN Protein

Six 'a'-position residues in the HRs were mutated to alanine (L74A, V81A, V88A, L96A, I103A, and L110A) and four asparagine-to-alanine mutants in the glycosylation sites (N119A, N341A, N433A, and N481A) were created by site-directed mutagenesis. Alanine was chosen because it has a short side chain and therefore should not disrupt a potential structure (fig. 1).

\section{Mutations in the HRs of the NDV HN Protein: CSE, Function, and Co-IP Assay}

To probe the role of the HRs in fusion promotion, all six HR-mutated HN proteins to complement the homologous $\mathrm{F}$ protein in the promotion of fusion were quantitated by the modification of the reporter gene assay. $\mathrm{HN}$ proteins carrying the L74A, V81A, V88A, I103A, and L110A mutations showed significant reductions in fusion 


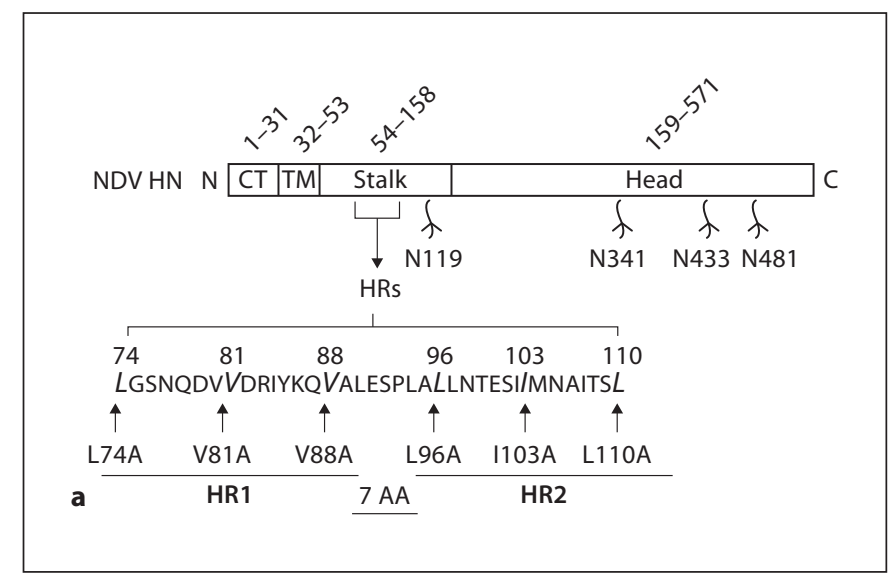

Fig. 1. Scheme of the locations of mutations. a Amino acid sequence of HRs showing residues 74-110 and location of glycosylation sites in the primary sequence of the NDV HN protein. Domain regions are indicated with NDV sequence numbers shown above. HR1 and HR2 are indicated with a line. The seven-aminoacid sequence (7 AA) between the HRs is denoted by a line below the sequence. Mutated residues are indicated in bold and italics with arrows. $\mathbf{b}$ The three-dimensional structure of the NDV AV$\mathrm{HN}$ and locations of mutated residues. A side view of the $\mathrm{HN}$ dimer with a two-helix bundle stalk is shown. The two monomers in the dimmer are shown in ribbon mode in magenta and cyan. The two helix bundle molecules are shown in sky blue and yellow tint. Mutated residues are shown in space-filling mode in one of the monomers. Residues 341, 433, and 481 are shown in blue. Residues $81,88,96,103$, and 110 are shown in green. Unfortunately, L74 and N119 were not provided in this structure. The figure was generated with RasMol, using the structures of Yuan et al. [32]. c Distribution of the residues of the HRs in the helical wheel representation. Six amino-terminal residues (L74, V81, V88, L96, I103, and L110) which were mutated to alanine are placed at position 'a' of the idealized helix and marked by the arrow.

activity, which was $22.6,29.9,24.7,9.1$, and $17.8 \%$ relative activity compared to that of the wt $\mathrm{HN}$, respectively (fig. 2a). The L96A mutation also resulted in approximately a reduction of one third in fusion promotion (70.4\% of wt $\mathrm{HN}$ activity). We qualitatively observed the extent of syncytium formation in monolayers of cells in which the weakly fusogenic HRs-mutant HN proteins were coexpressed with NDV F and stained for fusion. In contrast to vector-expressing cells, small syncytia can be seen in all six of the monolayers. In each case, the syncytia were not only small but also fewer than those in cells expressing wt $\mathrm{HN}$ and $\mathrm{F}$ (fig. 3).

To determine whether reduced fusogenic activity correlates with diminished capacity to interact with NDV F protein, the BHK-21 cells which coexpressed NDV HN
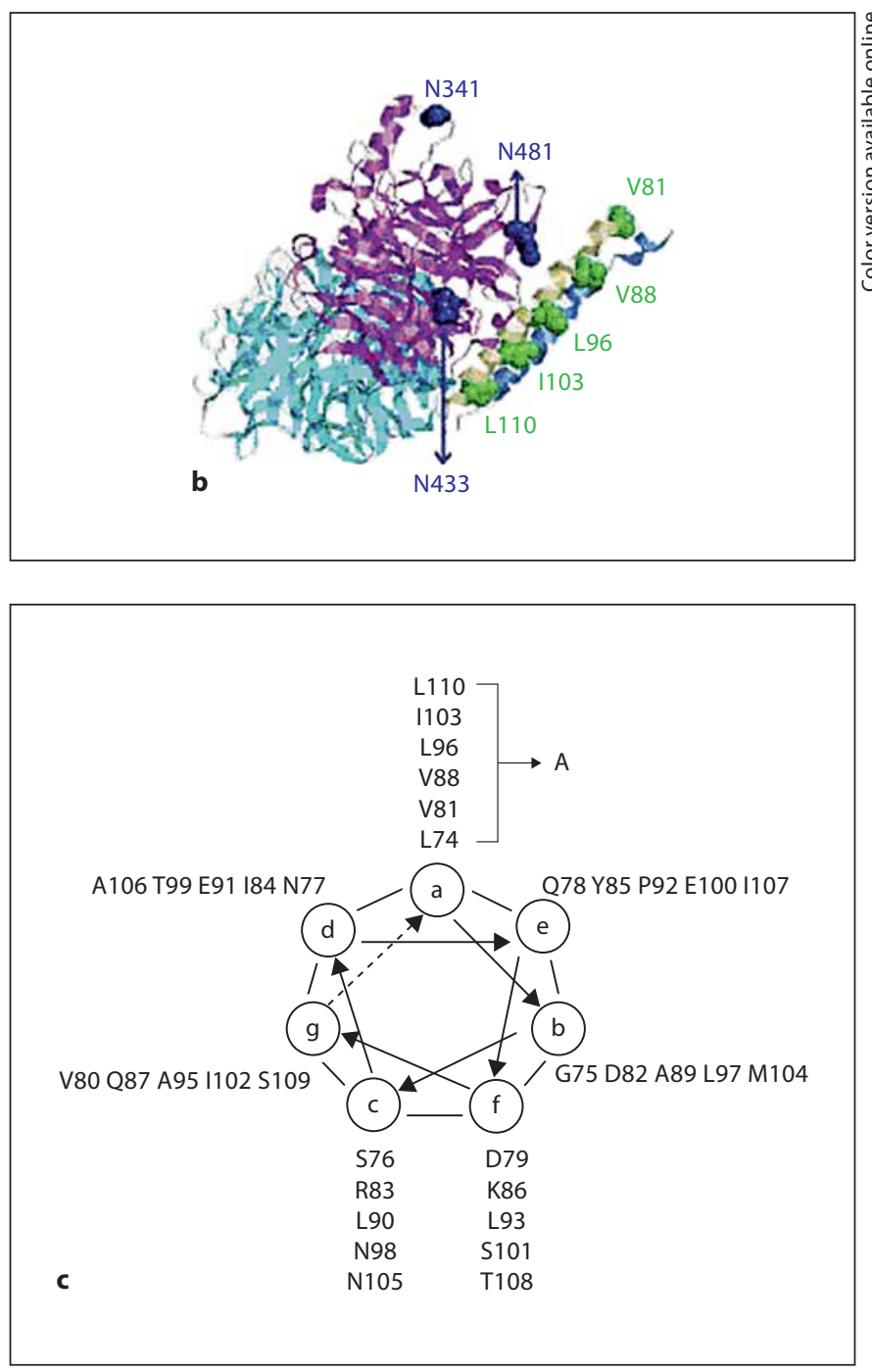

and Fcsm proteins were used to develop a co-IP assay (fig. $2 b-d)$. Fcsm was used to make sure that the extent of fusion does not affect the amount of $\mathrm{F}$ precipitable from the surface of the monolayer as described in Materials and Methods. Figure $2 \mathrm{~b}-\mathrm{d}$ showed the co-IP results for each of the HR-mutated HN proteins and critical controls. The first lane of each figure showed the maximum amounts of the two proteins that can be immunoprecipitated from the cell surface for each sample. The first pair of each figure showed that neither protein is present in control cells transfected with empty vector. The second pair of figures indicated that NDV HN did not immunoprecipitate with the anti-F MAb without coexpression of the NDV F protein, and the third pair demonstrated that the protein that coimmunoprecipitated with $\mathrm{F}$ was not present in cells in 

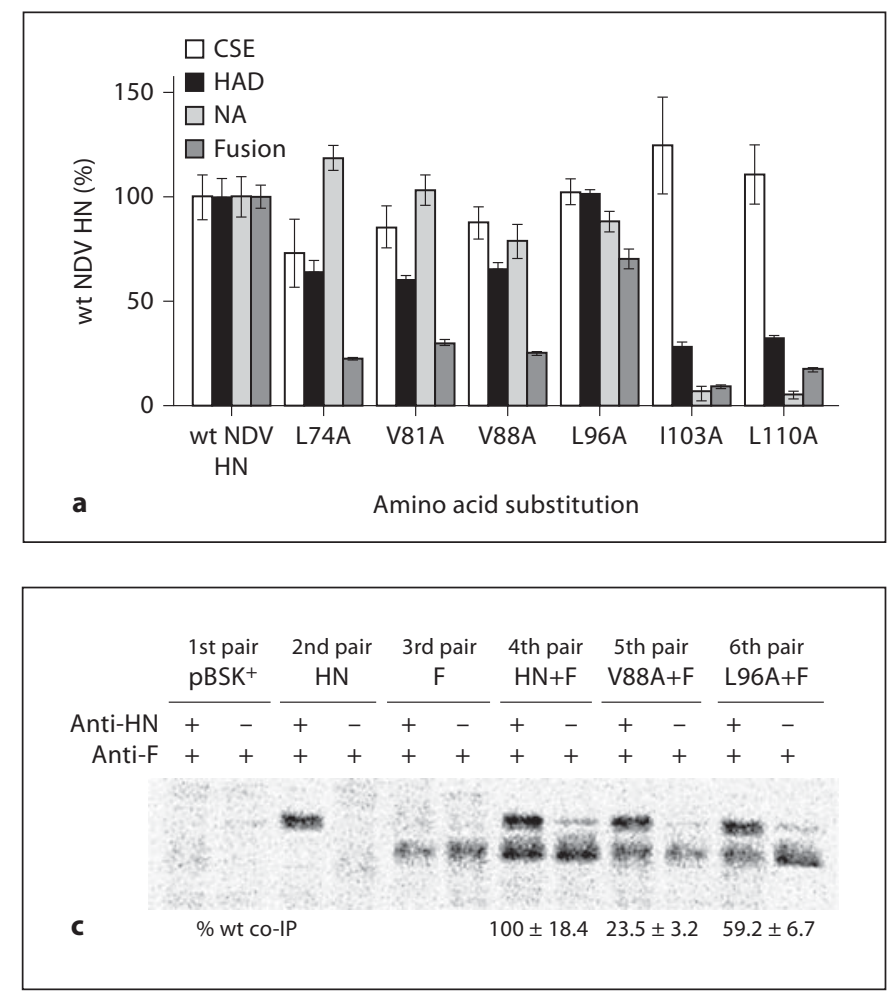

Fig. 2. CSE, functional characteristics, and co-IP assay of wt and mutated HN proteins in HRs. a Relative CSE efficiency and biological activities with mutations within HRs. CSE was quantitated by flow cytometry with a mixture of two MAbs specific for the NDV HN protein. The HAD activity was determined by the ability of $\mathrm{HN}$ protein to adsorb erythrocytes as described in Materials and Methods. The NA activity was determined colorimetrically using sialyllactose. For each assay, the background detected in cells transfected with vector had been subtracted. All data are represented as relative percentages of the activities of the mutants, with respect to wt HN treated in the same way. The results represent an average of three independent experiments; error bars represent the standard deviations. b Formation of complexes between L74A and V81A mutants of NDV HN with the homologous $\mathrm{F}$ protein at the cell surface was detected by co-IP assay. The surface proteins of transfected cells were biotinylated, and then the

the absence of the $\mathrm{HN}$ protein. These controls established the specificity of the co-IP of HN by the anti-F antibody under reducing conditions. The percentage of total cell surface $\mathrm{HN}$ that coimmunoprecipitated with $\mathrm{F}$ was 31.6 $\pm 5.8 \%$. However, no co-IP of HN could be detected for the I103A-mutated protein (fig. 2d), correlating with the extremely minimal fusogenic activity of this protein (fig. 2a). Although L110A-HN had a negligible fusion $(17.8 \pm 1.0 \%$ of the wt amount) at a level similar to I103Amutated protein, the mean of three determinations was $65.7 \pm 5.4 \%$ of the amount of wt $\mathrm{HN}$ at the cell surface
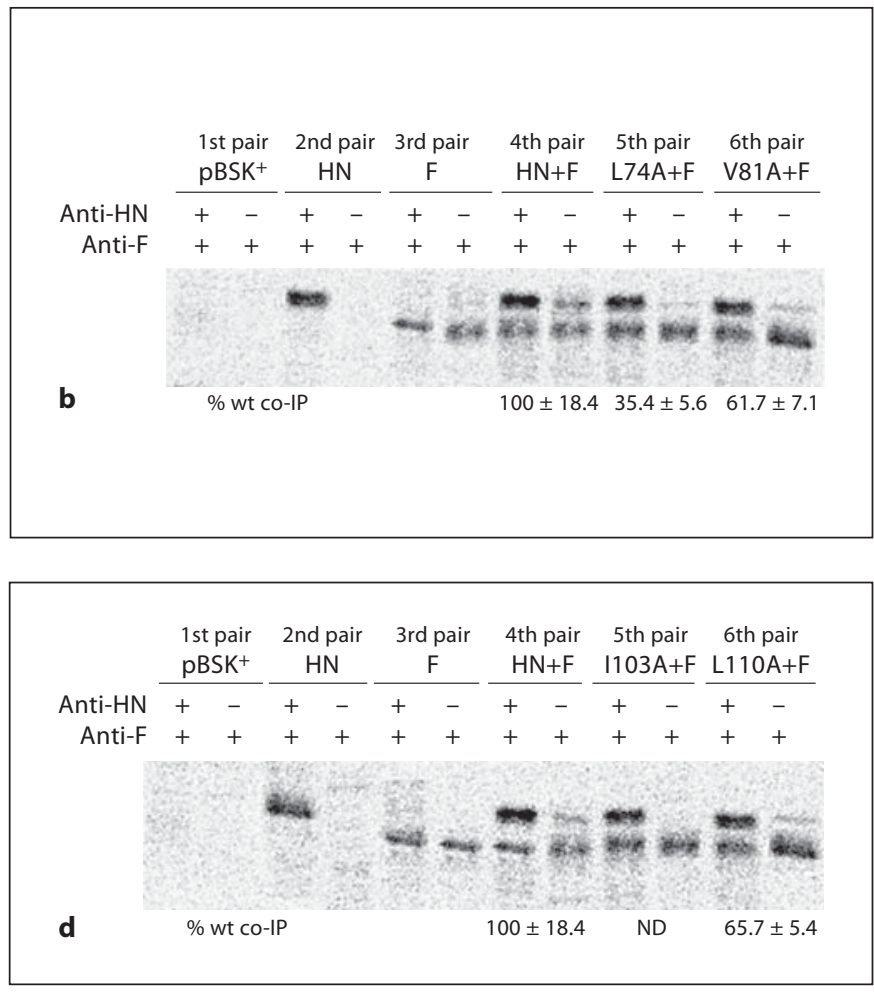

cells were lysed by PBS containing $1 \%$ Triton X-100, 0.5\% deoxycholate, and $1 \mathrm{mM}$ PMSF. The lysates were split into two equal aliquots and immunoprecipitated with either a mixture of an anti-F MAb and two anti-HN MAbs (the first lane in each pair) or an anti-F MAb alone (the second lane in each pair). The percentage of the total amount of the mutant HN protein at the cell surface that is coimmunoprecipitated with anti-F MAb is expressed compared to that of the wt HN. The results are expressed as mean \pm standard deviation for three independent experiments after normalization for differences in the expression of F. Immunoprecipitates were displayed on reducing SDS-polyacrylamide gels. + = Present; - = absent. c Co-IP of V88A- and L96A-mutated proteins with the homologous F protein. $\mathbf{d}$ Co-IP of proteins carrying a mutation at position 103 or 110 with the homologous $\mathrm{F}$ protein. $\mathrm{ND}=$ None detected.

coimmunoprecipitated with F (fig. 2d). L74A-, V81A-, and V88A-mutated proteins, which exhibited comparatively low fusion-promoting activities (ranging from 22.6 to $29.9 \%$ of the wt $\mathrm{HN}$ level), coimmunoprecipitated 35.4 , 61.7 , and $23.5 \%$ of the wt amount, respectively. Interestingly (fig. 2b, c), the L96A-mutated protein fused at $70.4 \%$ of the wt level and coimmunoprecipitated at $59.2 \%$ of the wt level (fig. 2c), less than that of L110A- and V81Amutated proteins. Thus, our data suggest that the amount of co-IP of $\mathrm{HN}$ is not directly proportional to the extent of fusion. 
Fig. 3. Cell fusion mediated by the mutants. BHK-21 cell monolayers were infected with recombinant vaccina virus and transfected with $\mathrm{pBSK}^{+}$and $\mathrm{pBSK}^{+}-\mathrm{F}$, $\mathrm{pBSK}^{+}-\mathrm{HN}$ and $\mathrm{pBSK}^{+}-\mathrm{F}$, or mutant $\mathrm{HN}$ cDNA and $\mathrm{pBSK}^{+}-\mathrm{F}$. At $24 \mathrm{~h}$ posttransfection, the monoplayers were fixed with methanol for $5 \mathrm{~min}$ and stained with Giemsa Accustain. Syncytia are indicated by arrows.

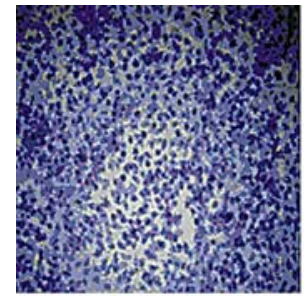

$\mathrm{pBSK}^{+}$

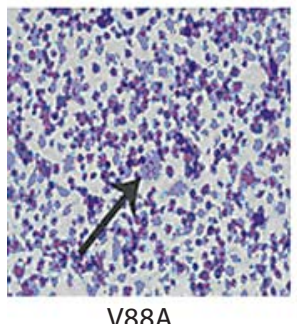

V88A

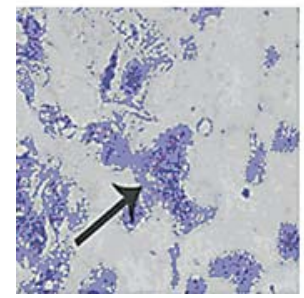

N119A

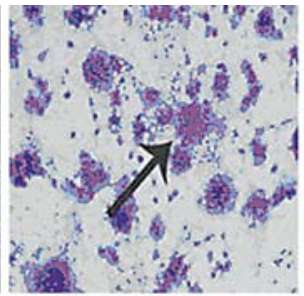

pBSK+-HN

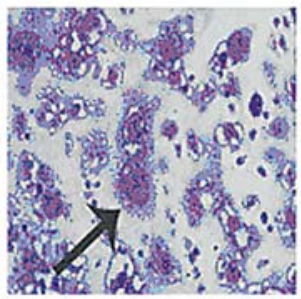

L96A

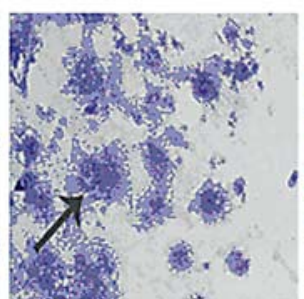

N341A
L74A
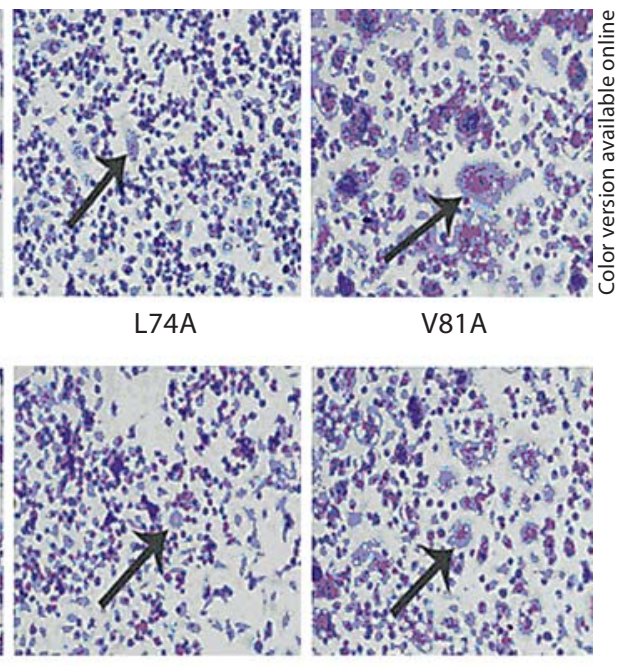

$1103 \mathrm{~A}$

L110A

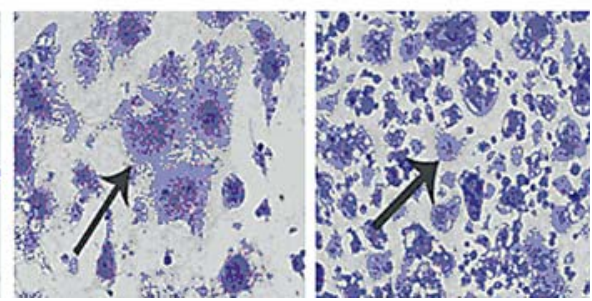

N433A
To determine the basis for the lack of fusion promotion activity by these mutated $\mathrm{HN}$ proteins, we have characterized their CSE and functions. The CSE of these mutated $\mathrm{HN}$ proteins was determined by flow cytometry with a mixture of two mouse anti-NDV MAbs (suppl. material fig. 2). As shown in figure 2a, each of the mutated proteins had a cell surface expression level similar to that of wt $\mathrm{HN}$, ranging from 72.7 to $124.4 \%$ of the wt $\mathrm{HN}$ level. L74Amutated protein and I103A-mutated protein were expressed at levels lower or higher than that of the wt $\mathrm{HN}$ level (72.7 and 124.4\%, respectively), which were not considered significantly different $(\mathrm{p}>0.05$, determined by $\mathrm{t}$ test). These results of near-wt levels of expression for HRsmutated proteins were also confirmed by co-IP studies.

The HAD activity of the HR-mutated HN proteins were determined by the ability to adsorb guinea pig erythrocytes at $4^{\circ}$ (suppl. material fig. 3). HN carrying L74A, V81A, or V88A substitution exhibited the slightly reduced HAD activity, which ranged from 59.9 to $65.4 \%$ of the wt level. Two of the amino acid substitutions, I103A and L110A, also significantly decreased receptor recognition to 28.2 and $32.3 \%$ of the wt level, respectively. However, the remaining L96A substitution exhibited the sim- ilar level of HAD activity to wt HN (fig. 2a). Therefore, the fusion deficiency of these mutated proteins except the L96A-mutated protein may be due to an effect on receptor recognition, but we cannot exclude the influence from the NA activity.

The NA activity was determined colorimetrically using sialyllactose. HN proteins carrying L74A, V81A, or L96A mutations had a similar NA activity to wt NDV HN. V88A-mutated HN still gained $78.5 \%$ of wt HN NA activity. However, the remaining mutants, I103A and L110A, exhibited levels of NA that were only 5.7 and 5.2\% of the wt amount, respectively. The weak NA activities of I103A-HN and L110A-HN were consistent with their significantly reduced fusion promotion activities (9.1 and $17.8 \%$ of the activity of wt HN, respectively) (fig. 2a).

\section{Mutations in the Glycosylation Sites of the NDV HN}

Protein: CSE, Function, IP, and Co-IP Assay

To determine the role of the removal of $\mathrm{N}$-linked carbohydrates in the biological activities of the HN protein and especially the ability to interact with F, we created four asparagine-to-alanine mutants in the glycosylation sites. To confirm that the mutated glycosylation sites ac- 

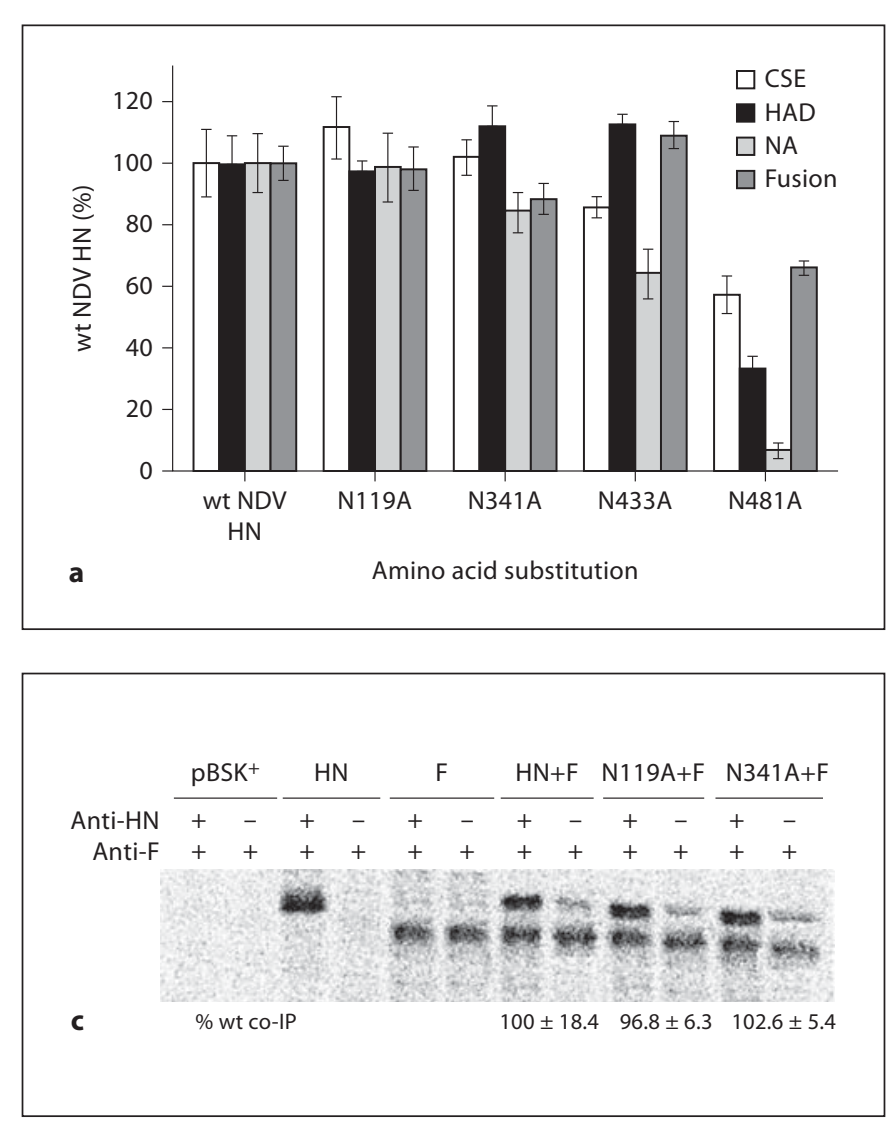

Fig. 4. CSE, functional characteristics, IP and co-IP assay of wt and mutations in the carbohydrate addition sites. a Relative CSE efficiency and biological activities with mutations in the carbohydrate addition sites. The assays were performed as described in the legend to figure $2 \mathrm{a}$. The data represent the means of three independent determinations plus standard deviations. b IP of $\mathrm{pBSK}^{+}$, wt or mutations in the carbohydrate addition sites. NDV wt and mutant HN proteins were expressed in BHK-21 cells, starved, labeled with Expre ${ }^{35} \mathrm{~S}^{35} \mathrm{~S}$ labeling mix for $3 \mathrm{~h}$ at $37^{\circ}$, and chased for $90 \mathrm{~min}$. The cells were lysed and the lysates were di-

tually lead to the loss of carbohydrates in the HN protein, we examined the expression and mobilities of the sites in a radioimmunoprecipitation assay. Radiolabeled cell lysates from BHK-21 cells expressing wt NDV HN or the G1-G4 N-glycan mutants were immunoprecipitated and analyzed on a $10 \%$ polyacrylamide gel to effectively visualize the relative mobilities of the labeled wt and mutant proteins. Our results showed that proteins with mutations in sites G1, G2, G3, and G4 (fig. 4b, lanes 3-6, respectively) migrated faster than wt protein and the PNGase F-digested mutant $\mathrm{HN}$ proteins comigrated with wt $\mathrm{HN}$ at a higher rate (fig. 4b, lanes 9-12, respectively), indicating that the altered migration rate of the mutated
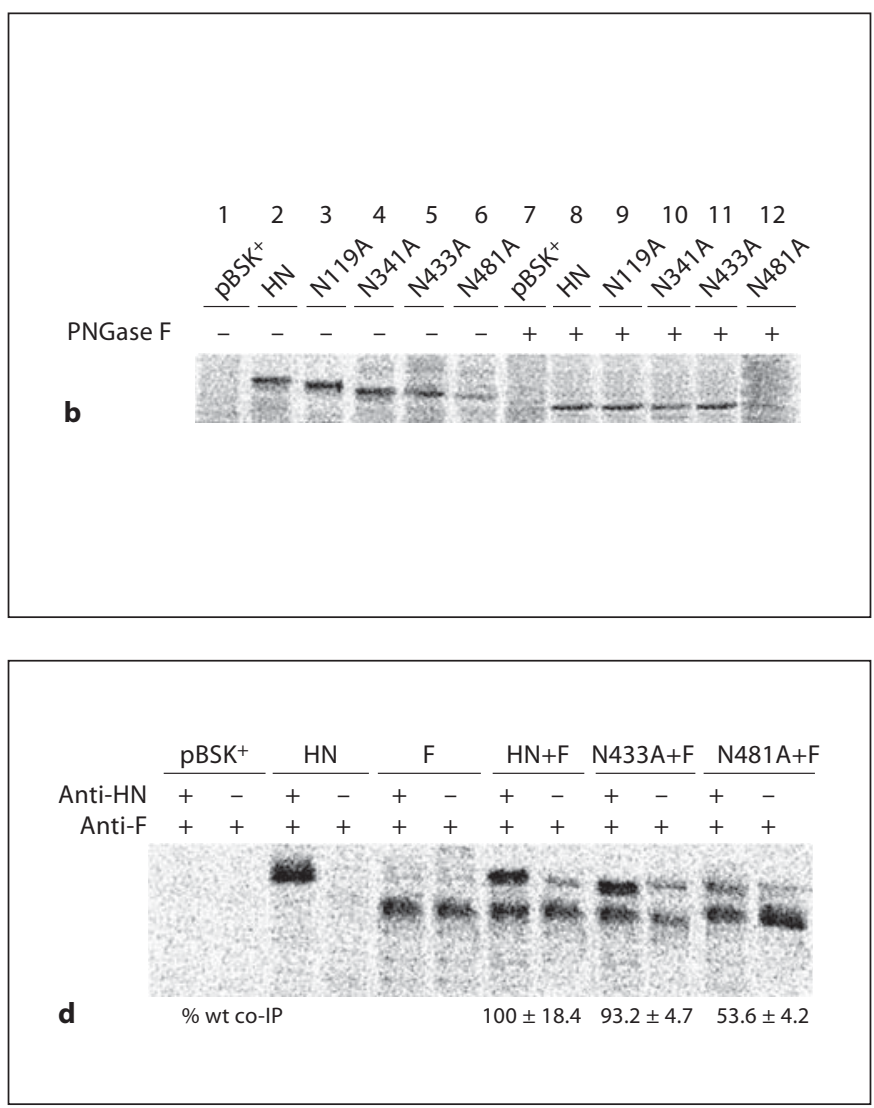

vided into two aliquots prior to IP of HN. After being washed, the immunoprecipitates were resuspended in PNGase F buffer. One aliquot was digested with $0.2 \mathrm{U}$ of PNGase F, and both aliquots were electrophoresed under reducing conditions. PNGase F cleaves the $\mathrm{N}$-glycan linkage of glycoproteins between asparagine and the carbohydrate chain. $+=$ Present; $-=$ absent. c Co-IP of N119A- and N341A-mutated proteins with the homologous F protein. The experiment was performed and the data were expressed as described in the legend to figure $2 \mathrm{~b}$. d Co-IP of N433A- and N481A-mutated proteins with the homologous F protein.

$\mathrm{HN}$ proteins is due solely to the loss of the different $\mathrm{N}$ linked carbohydrate chains. Furthermore, the G1 mutant migrated slightly slower than the other mutants, possibly because carbohydrate chains linked to N119 are smaller than those linked to other sites. Figure $4 \mathrm{~b}$ also showed that the amount of N481A-mutated HN protein was extremely small. This suggests an oligosaccharide at amino acid 481 of the NDV HN protein may have a significant effect on maturation and folding.

Next, the effects of the missed N-glycans on the ability of $\mathrm{HN}$ to complement $\mathrm{F}$ in the promotion of fusion were determined by the modification of the reporter gene assay. N-linked glycans at N119, N341, and N433 from 
$\mathrm{HN}$ protein still had similar fusion activity to the wt level. However, the HN protein carrying the N481A substitution gained only $66.2 \%$ ability to promote fusion compared to wt HN. It seems to be related to the diminished amount of N481A-HN determined by IP assay. To quantitate the expression level of the N481A-mutated protein the CSE of it was only $57.5 \%$ of the wt HN level. The other three mutations in the glycosylation sites were expressed at the near-wt level (fig. 4a).

We used a co-IP assay with the same mixture of antiHN MAbs for flow cytometry to determine whether N481A-HN reduced fusogenic activity by interfering with the HN-F interaction. The results showed that 53.6 $\pm 4.2 \%$ of the total amount of the mutant at the cell surface coimmunoprecipitated with F (fig. 4d). Figure $4 \mathrm{c}$ and $\mathrm{d}$ also showed that the remaining three mutant proteins coimmunoprecipitated with $\mathrm{F}$ at the near-wt level. Consequently, these results suggest that the immature and misfolding G4 mutant proteins have some effects on its interaction with the $\mathrm{F}$ protein.

The results presented above showed that the HN protein missing oligosaccharides at position 4 affects the formation of a mature molecule. However, proteins missing oligosaccharides at position 1,2 and 3 individually have no impairment in the formation of antigenic sites or CSE. To explore the role of these oligosaccharides in the biological activities of the HN protein, we performed an HAD and NA assay. The HAD activity and NA activity for each mutant were shown in figure $4 \mathrm{a}$. The $\mathrm{HN}$ protein carrying the N481A substitution had very low HAD (33.4\%) and NA $(6.5 \%)$ relative to the level of wt. The N119A-mutated and N341A-mutated HN proteins had similar HAD and NA activities compared to the wt. The N433A-HN had similar HAD activity similar to the wt (109.3\%), but had slightly lower NA activity (70.0\%) than wt.

Taken together, the results of the experiments for the N481 A mutants indicate that both deficient biological activities and the diminished ability to interact with F correlated with the malformation of a correct folding and mature molecule that the protein lacking glycosylation of G4 resulted in. However, mature proteins lacking glycosylations at positions 1, 2 and 3 individually had little or no impairment in their CSE and functions.

\section{Discussion}

The promotion of membrane fusion by most paramyxoviruses including NDV requires $\mathrm{HN}$ and $\mathrm{F}$ proteins derived from homologous viruses, suggesting that there is a specific and necessary interaction between the two proteins for fusion activation. However, the mechanism of HN-F protein-protein interaction is not clear. At present, two models for HN-F interaction have been postulated; one model suggests that attachment of $\mathrm{HN}$ protein to receptors stimulates the interaction of $\mathrm{HN}$ protein with $\mathrm{F}$ protein, resulting in $\mathrm{F}$ protein refolding to its active form. Another model proposes that $\mathrm{HN}$ and F proteins form a complex prior to $\mathrm{HN}$ protein attachment to $\mathrm{F}$ protein and that $\mathrm{HN}$ protein plays a role in the maintenance of the metastable conformation of the F protein. Studies of chimeric paramyxovirus HN proteins have shown that the presumed stalk domains of homologous $\mathrm{HN}$ proteins represent specificity for the F protein [1113]. Clearly, heptad repeats are able to interact with other heptad repeats by forming coiled coils, which are involved in protein-protein interactions [33]. Thus, we investigated the effect of mutations in a region containing two heptad repeats in the presumed stalk domain of the NDV HN protein (amino acids 74-110) and the ability to interact with the homologous $\mathrm{F}$ protein. Melanson and Iorio [23] previously reported that addition of $\mathrm{N}$-glycans in the stalk of the NDV HN protein blocks its interaction with the F protein and prevents fusion. Previous studies also showed that loss of $\mathrm{N}$-glycans in the NDV HN protein affects folding, activities of the protein and virulence of NDV. However, whether oligosaccharides have any effect on formation of HN-F protein complexes were not explored in these studies $[24,25]$. In this study, we tested the effects of lacking glycosylations of $\mathrm{HN}$ protein on $\mathrm{fu}$ sion and formation of $\mathrm{HN}-\mathrm{F}$ protein complexes to further elucidate the mechanisms of fusion and HN-F interaction.

Here, we generated six mutants in the 'a'-position residues to elucidate the mechanism by which the HRs of the $\mathrm{HN}$ protein may contribute to fusion and HN-F interaction. Our results showed that all the mutations in this region not only severely decreased HN-F fusion, but also interfered with its interaction with $\mathrm{HN}$ and $\mathrm{F}$ protein. All of the mutant HN proteins appeared to fold correctly and were still efficiently precipitated by two anti-HN MAbs compared to the wt HN protein. The L96A-mutated protein exhibited the same levels of HAD activity and NA activity as the wt protein, but has been coimmunoprecipitated at only $59.5 \%$ of the wt level and its fusion activity was only $70.4 \%$ of the wt level. This suggests that the substitution for L96 affects the fusion promotion function of $\mathrm{HN}$ by interfering with its interaction with the $\mathrm{F}$ protein. Mutations of the first two residues, 74 or 81 , did cause a decrease in receptor recognition to 63.8 and $59.9 \%$ 
of the wt level, respectively. They also resulted in the significantly similar loss of fusion promotion activity $(22.6$ and $29.9 \%$, respectively) relative to that of the wt $\mathrm{HN}$ protein, in spite of their co-IP with a reduced amount of NDV F (35.4 and 61.7\%, respectively). This supports the idea that the promotion of membrane fusion by NDV is dependent on the recognition of receptors by $\mathrm{HN}$ [21]. V88A-HN, I103A-HN, and L110A-HN have the same phenotype that is different from the other three mutations as described above. They decreased fusion promotion and the HN-F interaction and they also affected HAD and NA activity. Thus, it is most likely that the fusion-related defects in these substitutions are the result of an alteration of the conformation of globular domain that is responsible for receptor binding as well as NA activity. Furthermore, a signal being altered from the globular domain was transmitted to the F-specific region in the stalk, and thereby interfered with the HN-F interaction.

Again, we have explored the possibility whether differences in the HN-F interaction are consistent with differences in fusion promotion. However, the amount of co-IP of $\mathrm{HN}$ is disproportional to the extent of fusion. L74A$\mathrm{HN}, \mathrm{V} 81 \mathrm{~A}-\mathrm{HN}, \mathrm{V} 88 \mathrm{~A}-\mathrm{HN}$, and L110A-HN coimmunoprecipitated at different levels less than that of wt $\mathrm{HN}$ $(35.4,61.7,23.5$, and $65.7 \%$, respectively), but fused at approximately $25 \%$ of the wt level. These findings are inconsistent with a previous study, which proposed a direct correlation between loss of fusion and decreased detection of the NDV HN-F complex [15]. In fact, conditions which favor this are the fact that HAD and NA activity of mutant proteins is not affected or affected slightly, but mutant proteins except the L96A protein in this study more or less lost HAD and/or NA activity.

The role of deglycosylation in the folding and activities of $\mathrm{HN}$ is consistent with previous findings [24, 25]. The missing oligosaccharide at position 4 affected the formation of a mature molecule, activities, and HN-F interaction. Oligosaccharides at position 1,2, and 3 have little or no effect on the folding and function of NDV HN protein. These results suggest that elimination of oligosaccharides at position 4 have a great effect on maturation and folding of HN protein. This nascent and misfolding G4-mutated protein seems to alter the structure of the presumed globular head and F-specific region in the stalk, and thereby impair HAD activity, NA activity, and HN-F interaction, all of which are important for membrane fusion.

In summary, we have shown that mutations in the HRs that decreased HAD activity and/or NA activity also abolished its ability to interact with the homologous $\mathrm{F}$ protein at the cell surface. This can provide evidence for the first model indicating that the attachment of $\mathrm{HN}$ protein to receptors stimulates an interaction of $\mathrm{HN}$ protein with $\mathrm{F}$ protein. Also, we identified a point mutation (N481A) in the glycosylation sites that resulted in an impairment in folding and reduced the ability to interact with the homologous F protein at the cell surface and the promotion fusion activity. The ability of the latter to complement $\mathrm{F}$ in fusion is still dependent on its ability to bind receptors and NA activity. Thus, the N481A mutation indirectly affects the fusion promotion function of $\mathrm{HN}$ and $\mathrm{HN}-\mathrm{F}$ interaction by misfolding the important structure, while mutations in the HRs do that by altering the transmission of a signal from the globular domain to the Fspecific region in the stalk.

\section{Acknowledgements}

We gratefully acknowledge Dr. Xue-jie Yu and Dr. Li-li Chen for critical reading of the manuscript. We also thank Dr. Ronald M. Iorio for providing the recombinant plasmid vectors and Dr. Bernard Moss for vTF7-3.

This work was supported by a grant from National Natural Science Foundation of China (No. 30970142) and the Scientific Foundation of Innovative Research Team at the Shandong University, Jinan, People's Republic of China.

\section{References}

1 Iorio RM, Mahon PJ: Paramyxoviruses: different receptors - different mechanisms of fusion. Trends Microbiol 2008;16:135-137.

$\checkmark 2$ de Leeuw OS, Guus Koch, Leo Hartog, Ravenshorst N, Peeters Ben PH: Virulence of Newcastle disease virus is determined by the cleavage site of the fusion protein and by both the stem region and globular head of the haemagglutinin-neuraminidase protein. J Gen Virol 2005;86:1759-1769.
3 McGinnes LW, Pantua H, Laliberte JP, Gravel KA, Jain S, Morrison TG: Assembly and biological and immunological properties of Newcastle disease virus-like particles. J Virol 2010;84:4513-4523.

4 Moscona A: Entry of parainfluenza virus into cells as a target for interrupting childhood respiratory disease. J Clin Invest 2005; 115:1688-1698.
5 Mahon PJ, Mirza AM, Musich TA, Iorio RM: Engineered intermonomeric disulfide bonds in the globular domain of Newcastle disease virus hemagglutinin-neuraminidase protein: implications for the mechanism of fusion promotion. J Virol 2008;82:10386-10396.

6 Takimoto T, Taylor GL, Connaris HC, Crennell SJ, Portner A: Role of the hemagglutininneuraminidase protein in the mechanism of paramyxovirus-cell membrane fusion. J Virol 2002;76:13028-13033. 
7 Ren G, Wang Z, Wang G, Song Y, Yao P, Xu $\mathrm{H}$, Wen $\mathrm{H}$, Zhang W: Effects of heptad repeat regions of $\mathrm{F}$ protein on the specific membrane fusion in paramyxoviruses. Intervirology 2006;49:299-306.

8 Krishnan A, Verma SK, Mani P, Gupta R, Kundu S, Sarkar DP: A histidine switch in hemagglutinin-neuraminidase triggers paramyxovirus-cell membrane fusion. J Virol 2009;83:1727-1741.

-9 Tsunekuni R, Ito H, Kida H, Otsuki K, Ito T: Increase in the neuraminidase activity of a nonpathogenic Newcastle disease virus isolate during passaging in chickens. J Vet Med Sci 2010;72:453-457.

$>10$ Gravel KA, Morrison TG: Interacting domains of the $\mathrm{HN}$ and F proteins of Newcastle disease virus. J Virol 2003;77:11040-11049.

11 Deng R, Wang Z, Mirza AM, Iorio RM: Localization of a domain on the paramyxovirus attachment protein required for the promotion of cellular fusion by its homologous fusion protein spike. Virology 1995;209:457469.

12 Tanabayashi K, Compans RW: Functional interaction of paramyxovirus glycoproteins: identification of a domain in Sendai virus HN which promotes cell fusion. J Virol 1996; 70:6112-6118.

13 Tsurudome M, Kawano M, Yuasa T, Tabata $\mathrm{N}$, Nishio M, Komada H, Ito Y: Identification of regions on the hemagglutinin-neuraminidase protein of human parainfluenza virus type 2 important for promoting cell fusion. Virology 1995;213:190-203.

14 Stone-Hulslander J, Morrison TG: Mutational analysis of heptad repeats in the membrane-proximal region of Newcastle disease virus HN protein. J Virol 1999;73:36303637.

15 Melanson VR, Iorio RM: Amino acid substitutions in the F-specific domain in the stalk of the Newcastle disease virus HN protein modulate fusion and interfere with its interaction with the F protein. J Virol 2004;78: 13053-13061.
16 Sergel-Germano T, McQuain C, Morrison TG: Mutations in the fusion peptide and heptad repeat regions of the Newcastle disease virus fusion protein block fusion. J Virol 1994;68:7654-7658.

17 Buckland R, Maivoisin E, Beauverger P, Wild F: A leucine zipper structure present in the measles virus fusion protein is not required for its tetramerization but is essential for fusion. J Gen Virol 1992;73:1703-1707.

18 Reitter JN, Sergel T, Morrison TG: Mutational analysis of the leucine zipper motif in the Newcastle disease virus fusion protein. J Virol 1995;69:5995-6004.

19 Wang Z, Iorio RM: Amino acid substitutions in a conserved region in the stalk of the Newcastle disease virus HN glycoprotein spike impair its neuraminidase activity in the globular domain. J Gen Virol 1999;80:749753.

20 Deng R, Wang Z, Mahon PJ, Marinello M, Mirza A, Iorio RM: Mutations in the Newcastle disease virus hemagglutinin-neuraminidase protein that interfere with its ability to interact with the homologous F protein in the promotion of fusion. Virology 1999; 253:43-54.

21 Li J, Quinlan E, Mirza A, Iorio RM: Mutated form of the Newcastle disease virus hemagglutinin-neuraminidase interacts with the homologous fusion protein despite deficiencies in both receptor recognition and fusion promotion. J Virol 2004;78:5299-5310.

22 Mcginnes L, Sergel T, Reitte J, Morrison TG: Carbohydrate modifications of the NDV fusion protein heptad repeat domains influence maturation and fusion activity. Virology 2001;283:332-342.

23 Melanson VR, Iorio RM: Addition of N-glycans in the stalk of the Newcastle disease virus $\mathrm{HN}$ protein blocks its interaction with the $\mathrm{F}$ protein and prevents fusion. J Virol 2005;80:623-633.

24 Panda A, Elankumaran S, Krishnamurthy S, Huang Z, Samal SK: Loss of N-linked glycosylation from the hemagglutinin-neuraminidase protein alters virulence of Newcastle disease virus. J Virol 2004;78:4965-4975.
25 Mcginnes LW, Morrison TG: The role of individual oligosaccharide chains in the activities of the HN glycoprotein of Newcastle disease virus. Virology 1995;212:398-410.

-26 Zhang W, Ren G, Wu B, Liu X, Wang G, Song $\mathrm{Y}, \mathrm{Xu} \mathrm{H}$, Wen H, Wang Z: Amino acid substitutions in leucine zipper motif in the F-specific domain of human parainfluenza virus 3 $\mathrm{HN}$ protein play important roles in the protein function. Intervirology 2008;51:311-321.

27 Fuerst TR, Niles EG, Studier W, Moss B: Eukaryotic transient-expression system based on recombinant vaccinia virus that synthesizes bacteriophage T7 RNA polymerase. Proc Natl Acad Sci USA 1986;83:8122-8126.

28 Mirza AM, Deng R, Iorio RM: Site-directed mutagenesis of a conserved hexapeptide in the paramyxovirus hemagglutinin-neuraminidase glycoprotein: effects on antigenic structure and function. J Virol 1994;68: 5093-5099.

-29 Corey EA, Mirza AM, Levandowsky E, Iorio RM: Fusion deficiency induced by mutations at the dimer interface in the Newcastle disease virus hemagglutinin-neuraminidase is due to a temperature-dependent defect in receptor binding. J Virol 2003;77:6913-6922.

30 Aminoff BD: Methods for the quantitative estimation of $\mathrm{N}$-acetylneuraminic acid and their application to hydrolysates of sialomucoids. Biochem J 1961;81:384-392.

-31 Wang Z, Mirza AM, Li J, Mahon PJ, Iorio RM: An oligosaccharide at the C-terminus of the F-specific domain in the stalk of the human parainfluenza virus 3 hemagglutininneuraminidase modulates fusion. Virus Res 2004;99:177-185.

-32 Yuan P, Swanson KA, Leser GP, Paterson RG, Lamb RA, Jardetzky TS: Structure of the Newcastle disease virus hemagglutininneuraminidase $(\mathrm{HN})$ ectodomain reveals a four-helix bundle stalk. Proc Natl Acad Sci USA 2011;108:14920-14925.

33 Chambers P, Pringle CR, Easton AJ: Heptad repeat sequences are located adjacent to hydrophobic regions in several types of virus fusion glycoproteins. J Gen Virol 1990;71: 3075-3080 